\title{
Spatial Analysis when the Location of Infection is Uncertain: An Innovative Approach Using an Animal- Herd-Level Weighted Analysis
}

\author{
Céline Dupuy*1,2, Claire Morlot ${ }^{3}$, Pierre Demont ${ }^{4}$, Marie-Pierre Callait-Cardinal ${ }^{4}$, Christian \\ Ducrot $^{2}$, Didier Calavas ${ }^{1}$ and Emilie Gay ${ }^{1}$
}

${ }^{1}$ ANSES, Lyon, France; ${ }^{2} I N R A$, Saint Genès Champanelle, France; ${ }^{3}$ Direction générale de l'Alimentation, Paris, France; ${ }^{4}$ VetAgroSup, Marcy l'Etoile, France

\begin{abstract}
Objective
Spatial analysis of infectious diseases enables identification of areas at high risk for infection, a useful tool for implementation of risk-based surveillance. For chronic diseases, the period between infection and detection can be long and when animal movements are important, identifying the place of infection is difficult. The objective of this study is to propose an innovative approach for spatial analysis that takes into account uncertainty regarding the location where animals were infected. An animal-herd-level weighted analysis was used and applied to bovine cysticercosis in France.

\section{Introduction}

Bovine cysticercosis is a zoonotic foodborne disease caused by Taenia saginata involving cattle as the intermediate host and humans as the final host. Humans are infected by eating raw or undercooked meat of infected cattle. Cattle are infected after grazing on pasture infected by human feces. Disease detection in cattle is performed during post-mortem meat inspection at the slaughterhouse through the identification of cysts in muscle tissue. Cysts develop from a viable stage to a degenerated stage in one to nine months, both stages being visible and distinguishable in cattle muscle. Due to the slow development of cysts and the complexity of cattle movements (up to ten different herds from birth to slaughter in France), there is a strong bias to consider the last farm location before slaughter as the location of infection.
\end{abstract}

\section{Methods}

A spatial analysis was performed using a spatial scan statistic [2]. The background population was defined as the number of cattle slaughtered in each commune (French administrative area). The case population was defined as the number of animal-herd weights aggregated by commune. Animal-herd weight was defined as the probability that the animal was infected in a given herd. For instance, an animal that spent time in three different herds from birth to slaughter was assigned three probabilities to have been infected in one of these herds: $\mathrm{P}_{1}, \mathrm{P}_{2}$ and $\mathrm{P}_{3}\left(\mathrm{P}_{1}+\mathrm{P}_{2}+\mathrm{P}_{3}=1\right)$. Based on the chronology of cyst development, rules to attribute these probabilities have been defined. The model was adjusted for age and sex, since these variables are associated with bovine cysticercosis [1].

\section{Results}

The data used came from a national survey conducted in France in 2010 on all the cattle slaughterhouses and from the national cattle database for cattle movements. After removal of missing data, information regarding 4,557,593 cattle slaughtered in 181 slaughterhouses was used $(91.1 \%$ of cattle slaughtered in France in 2010). Among these, post mortem inspection allowed the identification of 6,431 cattle harboring at least one cyst (i.e. regardless of its stage of development) and 603 harboring at least one viable cyst. Three significant clusters for cattle with all types of cysts were detected (Figure A). Only one significant cluster was detected in eastern France when cattle with viable cysts only were taken into account (Figure B).

\section{Conclusions}

Formerly, only cattle with viable cysts, i.e. acute lesions, would have been considered for spatial analysis so as to limit bias regarding the location of infection. The difference in location of the clusters detected, when considering only cattle harboring viable cysts or cattle harboring all types of cysts, proved the relevancy of this novel approach. Animal-herd-level analysis could be useful for other chronic diseases, such as bovine tuberculosis, for which the period between infection and detection can be long and variable. Dedicated rules to attribute probabilities taking into account the evolution of the disease would then have to be defined.

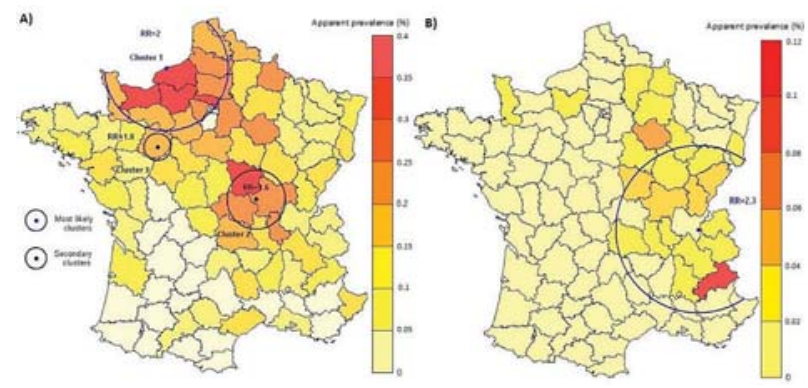

Apparent prevalence (\%) of bovine cysticercosis (A: with viable or degenerated cysts; B: with viable cysts only) and significant clusters of cattle harboring viable or degenerated cysts (A) and viable cysts only (B). $R R=$ relative risk.

\section{Keywords}

Animal health surveillance; Public Health; Spatial analysis; zoonosis

\section{References}

1.Dupuy, C., et al. (2014). Construction of standardized surveillance indicators for bovine cysticercosis. Prev Vet Med, 115, 208-292.

2.Kulldorff, M. (1997). A spatial scan statistic. Theory and Methods, 26(6), 1481-1496.

*Céline Dupuy

E-mail: celine.dupuy@agriculture.gouv.fr 